the development of arthritis or are rather a consequence of the inflammatory processes. Furthermore, while both germ-free condition and administration of oral antibiotics prevent arthritis in mice, it is unclear whether modulation of the intestinal microbiota after the onset of arthritis may still suppress the disease. Objectives: We aimed to assess potential alterations of the intestinal microbiome in the preclinical phase of inflammatory arthritis, and to determine the efficacy of microbiota modulations in the treatment of established disease in mice.

Methods: We sequenced fecal bacterial 16S rRNA to define the intestinal microbiome in mice before immunization with collagen and 21 days later before the booster injection for the induction of collagen-induced arthritis (CIA). To assess the efficacy of microbiota modulation during arthritis, mice with ongoing CIA were treated orally by a broad-spectrum antibiotic cocktail for one week to partially eliminate the intestinal microbiota. T cell differentiation and production of cytokines in intestinal lamina propria and joint-draining lymph nodes were assessed by flow cytometry and Luminex. Arthritis was assessed macroscopically and by histology. Serum-transfer arthritis induced by intraperitoneal injections of arthritogenic $\mathrm{K} / \mathrm{BxN}$ mouse serum was used as a control, T cell-independent, model.

Results: The preclinical phase of inflammatory arthritis in mice was characterized by marked changes in the intestinal microbiome, represented by a significant increase of the phylum Bacteroidetes and a decrease of Firmicutes and Proteobacteria. Among the most abundant bacterial families, S24-7 and Staphylococcaceae were expanded, whereas Lachnospiraceae were reduced during the immune priming phase of CIA. Several operational taxonomic units associated with S24-7 family increased, while those assigned to Lachnospiraceae and Ruminococcaceae decreased in the intestinal microbiota before the clinical onset of arthritis. The abundance of intestinal lamina propria Th17 cells significantly correlated with the severity of $\mathrm{ClA}$; however, intestinal Th1 cells were not correlated with the disease. Elimination of intestinal microbiota in mice with ongoing $\mathrm{CIA}$ specifically suppressed intestinal Th17 cell differentiation without affecting Th1 and Treg cells. Importantly, elimination of intestinal microbiota suppressed Th17 cell differentiation and IL-17 production in joint-draining lymph nodes, and reduced the severity of established CIA. In contrast, the T cell-independent serum-transfer arthritis was not affected by this strategy.

Conclusions: These observations suggest that perturbations of the intestinal microbiome may precede the development of inflammatory arthritis. Similar studies are warranted in human pre-RA or at-risk individuals to shed light on the functional role of the microbiome in the development of RA. Our studies also suggest that modulation of the intestinal microbiota after the onset arthritis may still provide opportunities to treat inflammatory arthritis.

Disclosure of Interest: None declared

DOI: 10.1136/annrheumdis-2017-eular.5533

\section{FRI0073 RAGE ACTIVITY INFLUENCES CO-DEVELOPMENT OF JOINT AND VASCULAR DISEASE IN RA}

S. Yu $^{1}$, L.-S. Tam ${ }^{2} .{ }^{1}$ Rheumatology, The Second Affiliated Hospital of Guangzhou Medical University, Guangzhou, China; ${ }^{2}$ Rheumatology, Medicine \& Therapeutics, Hong Kong, Hong Kong

Background: RAGE is expressed by many cells in blood and joints of RA and interacts with a variety of pro-inflammatory ligands, especially HMGB1 that are enriched in inflamed joint. The RAGE-ligand interaction leads to a sustained inflammatory response. Soluble RAGE (sRAGE) lacks the transmembrane and cytoplasmic domain of the cell surface full-length RAGE (fIRAGE) and function as a decoy for RAGE ligand. Relatively little is known about factors that regulate sRAGE levels in human subjects and whether circulating levels reflect tissue RAGE expression and activity is unclear. The relationship between the upregulation of fIRAGE/HMGB1 and the level of "protective" sRAGE levels in RA is of obvious clinical interest.

Objectives: To elucidate the balance between the expression of fIRAGE on peripheral blood monocyte and dendritic cell (DC), and the plasma concentration of SRAGE and HMGB1 in patients with active RA compare to controls; To ascertain whether fIRAGE expression profiles, plasma sRAGE and HMGB1 level correlated with disease activity or inflammatory markers in RA patients.

Methods: 40 consecutive patients attending the rheumatology clinic at Prince of Wales Hospital, who fulfilled the 2010 ACR/RULAR classification criteria with active RA (28 joint disease activity score 28-4 [CRP] (DAS28) $>3.2$ ) and 40 ageand sex-matched healthy volunteers were recruited for this cross-sectional study. The expression profile of cellular transmembrane RAGE on peripheral blood monocyte (ILT3 $\left.{ }^{+} \mathrm{CD} 123^{-}\right)$, total $\mathrm{DC}\left(\mathrm{ILT3}^{+} \mathrm{CD} 14^{-} \mathrm{CD} 16^{-}\right)$, myeloid DC $\left(\mathrm{ILT3}^{+} \mathrm{CD} 14^{-} \mathrm{CD} 16^{-} \mathrm{CD}^{\left.-23^{-}\right)}\right.$and plasmacytoid DC (ILT3 $\left.{ }^{+} \mathrm{CD} 14^{-} \mathrm{CD} 16^{-} \mathrm{CD} 123^{+}\right)$, plasma levels of HMGB1 and soluble RAGE in all RA patients and healthy controls were measured at baseline using flow cytometry and ELISA. In RA patients, associations with disease activity and severity variables were analyzed by simple and multiple linear regressions.

Results: Protein expression of fIRAGE on peripheral blood monocytes (ILT3 ${ }^{+} \mathrm{CD}_{123^{-}}$), total DCs (ILT3 ${ }^{+} \mathrm{CD} 14^{-} \mathrm{CD} 16^{-}$) and myeloid DCs (ILT3 $\left.{ }^{+} \mathrm{CD} 14^{-} \mathrm{CD} 16^{-} \mathrm{CD}^{-} 3^{-}\right)$were significant increased in RA patients with active disese compared with control subjects (all $p<0.01$ ) (no enough cells for fIRAGE detection on plasmacytoid ILT3 $\left.{ }^{+} \mathrm{CD} 14^{-} \mathrm{CD} 16^{-} \mathrm{CD} 123^{+} \mathrm{DCs}\right)$. Also, the fIRAGE was more common in patients with cardiac affection. There was no statistically significant difference of the plasma level of HMGB1 from active RA patients as compared healthy controls $(p>0.05)$. The plasma sRAGE level was significantly lower in patients compared to healthy controls $(\mathrm{p}<0.001)$, which correlated negatively with serum levels of CRP, ESR, DAS28 and high-density lipoprotein (HDL) (all $p<0.05$ ). Linear regrssion analysis detected CRP as significant predictors for sRAGE level.

Conclusions: Autoimmunity-mediated inflammation might induce aberrant expression and activation of fIRAGE while decreasing the plasma level of SRAGE in RA patients, subsequently leading to the augmented inflammatory response. Moreover, membrance fIRAGE and sRAGE were associated not just with RA inflammation and autoantibody protein, but also with classical vascular risk factors for end-organ damage. These data suggest that RAGE activity influences co-development of joint and vascular disease in RA patients.

Disclosure of Interest: None declared

DOI: 10.1136/annrheumdis-2017-eular.1701

\section{FRI0074 ANTI-INFLAMMATORY EFFECT OF RESVERATROL IN VITRO; POTENTIAL ROLE IN MANAGING LOW DISEASE ACTIVITY IN ARTHRITIS?}

S. Lomholt ${ }^{1}$, A. Mellemkjaer ${ }^{2}$, M.B. Iversen ${ }^{2}$, S.B. Pedersen ${ }^{3}$, T.W. Kragstrup ${ }^{4}$ ${ }^{1}$ Aarhus University, Aarhus C, Denmark; ${ }^{2}$ Department of Biomedicine, Aarhus University; ${ }^{3}$ Department of Endocrinology and Internal Medicine; ${ }^{4}$ Department of Rheumatology, Aarhus University Hospital, Aarhus C, Denmark

Background: Resveratrol (RSV), a non-toxic polyphenol found in grapes, certain nuts, roots etc., have received increased attention in the last decade due to its anti-inflammatory modulation of a number of pathways, including cyclooxygenase1/-2, nuclear factor kappa-beta and cytokine production. In vitro, RSV has been shown to reduce production of interleukin 1-beta and tumor necrosis factor alpha in monocytes and inhibit T-cell activation and synoviocyte proliferation. In vivo, intra-articular injections of RSV have demonstrated anti-inflammatory and pannus inhibiting effects in rats with induced arthritis.

Objectives: Here, we tested whether the anti-inflammatory effect of RSV in arthritis patients depends on the degree of systemic inflammation and the cellular composition of extracted synovial fluid. Furthermore, we evaluated the anti-inflammatory effect of RSV in combination with methotrexate (MTX) and adalimumab.

Methods: Synovial fluid mononuclear cells (SFMCs) from patient with rheumatoid arthritis ( $n=7)$ and spondyloarthritis ( $n=7$ ) were cultured in monoculture for 48 hours (in vivo activated lymphocytes and monocytes) or 21 days (spontaneous generation of osteoclasts). Cultures were either left untreated or treated with RSV $(25 \mu \mathrm{M})$, methotrexate $(0.5 \mu \mathrm{g} / \mathrm{ml})$, adalimumab $(5 \mu \mathrm{g} / \mathrm{ml})$ or in combination. Supernatants were analysed for the production of monocyte chemoattractant protein-1 (MCP-1) and matrix metalloproteinase 3 (MMP3). Osteoclast differentiation was analysed with a tartrate resistant acidic phosphatase (TRAP) enzymatic assay.

Results: In the SFMC cultures, RSV reduced MCP-1 production by $25 \%$ compared with untreated cells $(P=0.032)$. When grouping results by c-reactive protein (CRP), i.e. < median vs. $\geq$ median, the inhibitory effect of RSV was primarily seen in cultures from patients with CRP $<21 \mathrm{mg} / \mathrm{l}$. In this group, RSV reduced MCP-1 production by $63 \%$. Combining MTX and RSV reduced MCP-1 production compared to MTX alone, but only in the group of patients with CRP $<21 \mathrm{mg} / \mathrm{l}(\mathrm{P}=0.002)$. Combining adalimumab and RSV seemed to reduce MCP-1 in the group of patients with CRP $<21 \mathrm{mg} / \mathrm{l}$, but increase production in patients with CRP $\geq 21 \mathrm{mg} / \mathrm{l}(P=0.03)$. Similar grouping based on lymphocyte count showed RSV, MTX and adalimumab, alone or in combination, all reduced MCP-1 significantly compared to untreated cells in cultures from patients with $\geq 62 \%$ lymphocytes in the synovial fluid. RSV, MTX and adalimumab did not affect MMP3 production in the SFMC cultures. In the osteoclast cultures, RSV alone did not affect MCP-1 or TRAP. However, the combination of RSV and MTX reduced MCP-1 compared to no treatment $(P=0.004)$. Adalimumab alone or combined with RSV reduced TRAP compared with untreated cultures $(\mathrm{P}<0.027)$.

Conclusions: RSV exhibits an anti-inflammatory effect on SFMCs. Interestingly; our data suggest that this effect is most pronounced in patients with relatively low CRP. Further, RSV produces an additive anti-inflammatory response in combination with MTX in the group of patients with low CRP and a synovial fluid dominated by lymphocytes. Together, this suggests that RSV may possess an additive potential when added to MTX treatment of arthritis patients with low disease activity.

Disclosure of Interest: None declared

DOI: 10.1136/annrheumdis-2017-eular.2635

\section{FRI0075 RHEUMATOID ARTHRITIS PATIENTS RECEIVING GLUCOCORTICOID EXHIBITS SIGNIFICANT BONE QUALITY ABNORMALITIES, LEADING TO SEVERE BONE FRAGILITY REGARDLESS OF BISPHOSPHONATE TREATMENT}

T. Shimizu ${ }^{1,2}$, M. Takahata ${ }^{1}$, H. Hamano ${ }^{1}$, M. Ota ${ }^{1}$, S. Hiratsuka ${ }^{1}$, N. Iwasaki ${ }^{1}$ ${ }^{1}$ Department of Orthopeadic Surgery, Hokkaido University School of Medicine, Sapporo, Japan; ${ }^{2}$ Department of Radiology \& Biomedical Imaging, Musculoskeletal Quantitative Imaging Research, University of California San Francisco, San Francisco, United States

Background: Patients with rheumatoid arthritis (RA) receiving glucocorticoids 